EPJ Web of Conferences 52, 10002 (2013)

DOI: $10.1051 /$ epjconf/20135210002

(C) Owned by the authors, published by EDP Sciences, 2013

\title{
Some contributions of MAGIC to the physics of cosmic rays
}

\author{
S.R.Gozzini ${ }^{1, a}$ for the MAGIC Collaboration ${ }^{2}$ \\ ${ }^{1}$ Deutsches Elektronen-Synchrotron (DESY) Zeuthen, Germany \\ ${ }^{2}$ Observatorio del Roque de los Muchachos, La Palma, Spain
}

\begin{abstract}
Cosmic ray interactions can be investigated indirectly in $\gamma$ ray astronomy, with the observation of spectral and morphological features of certain classes of sources. MAGIC is a stereoscopic system of two $\gamma$ ray telescopes, located at La Palma (Canaries), with access to the energy window between $50 \mathrm{GeV}$ and $30 \mathrm{TeV}$. Sources of high relevance for the study of very high energy hadronic interactions are active galactic nuclei, as blazars and radio galaxies. MAGIC has detected about fifty such extragalactic objects; we will present some where the emission is explained with accelerated hadrons in interaction with ambient photons. We will also mention cosmic ray acceleration in galaxy clusters. Other than that, hadron-hadron interactions are supposed to take place in some supernova remnants in interaction with surrounding molecular clouds; we will show some results, in connection with cosmic rays of galactic origin. Finally, about other possible components, we will mention the measurement of the diffuse electron and positron spectrum. Trustingly, the close connection between particle physics and astrophysics will contribute in future years to many new interesting observations.
\end{abstract}

\section{Introduction}

This paper presents a sample of the results obtained with the MAGIC telescopes, focused on the connection between high energy $\gamma$-rays $(\mathrm{GeV}-\mathrm{TeV}$ regime, observed with Cherenkov telescopes) and their parent particles, which can be electrons, protons or nuclei accelerated to high energies. Electrons produce $\gamma$ rays mainly through inverse Compton scattering; hadrons radiate via two main channels of inelastic collisions: hadronic interactions of protons and nuclei on ambient molecular gas

$$
p N \rightarrow M^{0}+X \rightarrow \gamma+X,
$$

where $M^{0}$ is a neutral meson $\left(\pi^{0}, \eta\right)$, or via photo-meson production on ambient photons

$$
p \gamma \rightarrow \Delta \rightarrow \pi^{0}+X \rightarrow \gamma+X
$$

The cross section for $p N$ interactions is more than two orders of magnitude larger than for $p \gamma$, but the second process dominates in sources that have a high photon number density. Target baryons are found in the diffuse interstellar medium or in molecular clouds; seed photons belong to the ambient (cosmic microwave background, diffuse interstellar radiation field) or to the source itself (synchrotron radiation, accretion disk). For astrophysical applications, cross sections for hadronic processes are obtained with Monte Carlo simulations (SOPHIA, SIBYLL, QGSJET, [1-3]) and often presented in parameterized form [4-6]. Rates are obtained multiplying cross sections with target densities, and folding them with a spectrum of accelerated

\footnotetext{
a e-mail: rebecca.gozzini@desy.de
}

projectiles. Both densities and spectra are in principle unknown. The uncertainties on the computed photon spectra are currently dominated by large uncertainties on spectral parameters and quantities describing the environment. For the latter (such as target densities, magnetic field strength), in some cases independent measurements exist.

\section{The detection of $\gamma$ rays and the MAGIC telescopes}

Showers induced by photons in the atmosphere emit Cherenkov light that can be recorded by a photomultiplier camera; this is the method used by imaging atmospheric Cherenkov telescopes. These instruments perform 'traditional' astronomical observations, pointed at selected sources whose positions are known from observations at other energies. Photon arrival direction and energy are reconstructed to yield energy spectrum and morphology of the source observed, after filtering background of different origins (atmospheric hadrons, muons, night sky light, electronics). MAGIC is a stereo system of two dishes (17 metres diameter, $3.5^{\circ}$ field of view) located at La Palma, Canaries. The energy window accessible with this telescope ranges from $50 \mathrm{GeV}$ to about $30 \mathrm{TeV}$; at energies larger than $300 \mathrm{GeV}$, MAGIC has a sensitivity of $0.8 \%$ of the Crab flux in 50 hours, an angular resolution of $0.07^{\circ}$, and an energy resolution of $17 \%$. The two telescopes operate in stereo mode since 2009; an upgrade of camera and readout has been completed in summer 2012. Other technical details can be found in [7]. 


\section{Extragalactic sources and accelerated hadrons}

Active galactic nuclei (AGN) are thought to be principal accelerators of cosmic rays [8]. In these sources, a presumed super-massive black hole $\left(10^{7}-10^{9} M_{\odot}\right)$ powers the acceleration of particles through the formation of an active accretion disk that is thought to generate powerful, relativistic highly collimated jets. Hadronic models explain the observed emission by the interactions of protons with ambient photons of different origins: light from the accretion disk, or synchrotron radiation produced by electrons co-accelerated with protons in presence of magnetic field. In the simplest models, accelerated particles are confined inside a blob which moves coherently along the jet. In the blob, photons are radiated directly from decays of baryons or mesons and synchrotron radiation; electromagnetic cascades are initiated by all charged secondary particles produced in photo-hadronic interactions. Acceleration and emission features in AGNs are explained in a unified model [9], and the sources and their characteristics in different energy bands depend on the orientation of the jet relative to the observer. The optical depth inside the blob must be relatively small for photons to escape selfinteractions and reach the observer; the blob size is correlated with the time scale of variability (flares) observed in this class of sources.

\subsection{Monitored blazars: Markarian 421 and Markarian 501}

Blazars are all AGNs whose jet points in the direction of the observer. Markarian 421 and Markarian 501 are two AGNs observed with complete campaigns (several instruments and many years of coverage)[10,11]. Models of their spectral energy distribution, so far, cannot confirm nor exclude the acceleration of hadrons. Figure 1 shows a sample hadronic model hadronic model for Markarian 421 , which reproduces the emission with accelerated protons. The emission is due to photons from $\pi^{0}$ decay, and from cascades. Cascades are sequences of electromagnetic processes, initiated by $\pi^{0}$ decay, by electrons from pion and muon decays, and by synchrotron photons radiated from initial protons, or from any unstable intermediate charged particle $\left(\pi^{ \pm}, \mu^{ \pm}\right)$before decaying (see caption). For Markarian 501, the quiescent emission can be explained with leptons; however, flares have been interpreted with a change in the composition of accelerated particles [12].

\subsection{Radio galaxies: Perseus and Virgo Cluster}

Powerful radio lobes and large scale jets have been looked at as interesting accelerators in the quest for sources of cosmic rays [13]. Perseus is one of the brightest clusters in $\mathrm{X}$ rays; it contains two radio galaxies visible at very high energies: NGC 1275 and IC 310 (shown in the map in figure 2)[14]. The radiation of $\gamma$ rays is supposed to originate from cosmic ray interactions with the intracluster medium. Constraints on interactions between cosmic rays and the interstellar medium have been obtained from the non-detection of any $\gamma$ ray excess for NGC 1275 above $630 \mathrm{GeV}$. Limits have been established on the ratio between cosmic ray and thermal pressure (smaller than a few percent) and on the central magnetic field (larger than 4-9 $\mu \mathrm{G})[14]$.

The central active galaxy in the Virgo cluster, M87, has been rather discouraging from the perspective of the search for cosmic ray sources. Monitoring campaigns with MAGIC favour leptonic models for the low emission state [15]. Ejection events seen in multi-wavelength campaigns [16] are also interpreted with accelerated electrons emitting through synchrotron and inverse Compton processes.

\subsection{Flat spectrum radio quasars}

Flat spectrum radio quasars are a class of AGNs with a strong accretion disk, that forms an additional target photon field for $p \gamma$ interactions. These sources show atomic emission lines originating in the disk in what is called the "broad line region". It is believed that the $\gamma$-ray emission has a strong contribution from the interaction of the blob with a region characterized by an extremely high photon number density; this correlates also the size of the broad line region, and its distance from the central black hole, with the scale of variability. There is no definitive evidence that flat spectrum radio quasars are hadron accelerators, but many models hint in this direction. One example is the flat spectrum radio quasar 3 C 279, the most distant source detected at very high energy [17], whose emission is explained with proton and muon synchrotron and relative cascades [18]. The emission of other flat spectrum radio quasars is under debate. One of the latest discoveries in this regard is the source PKS 1222+216 (red shift $z=0.432$ ) [19]. The spectral energy distribution measured with Fermi-LAT and MAGIC measured with Fermi and MAGIC [19] are compatible with a simple power law

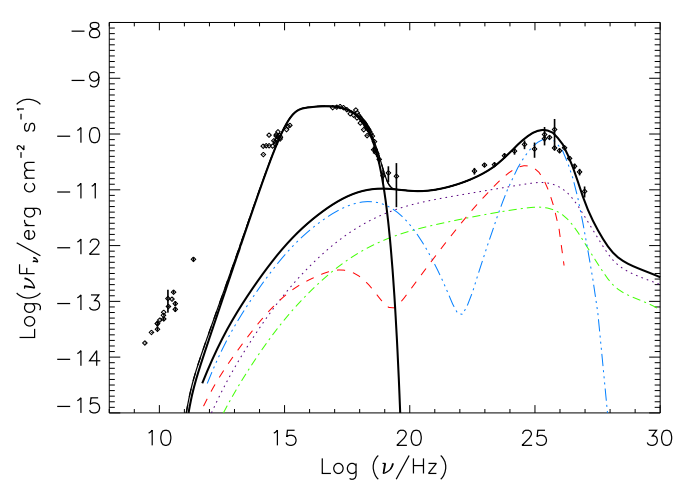

Figure 1. Spectral energy distribution of Markarian 421 interpreted with a hadronic model [10]. The fit has parameters: magnetic field $B=50 \mathrm{G}$, blob radius $=4 \cdot 10^{14} \mathrm{~cm}$ and an initial spectrum of protons following a power law with index -1.9. Cascades from $\pi^{0}, \pi^{ \pm}$, muon synchrotron and proton synchrotron are respectively marked as black dotted, green dashed-dotted, blue dashed-triple-dotted and red dashed line. 

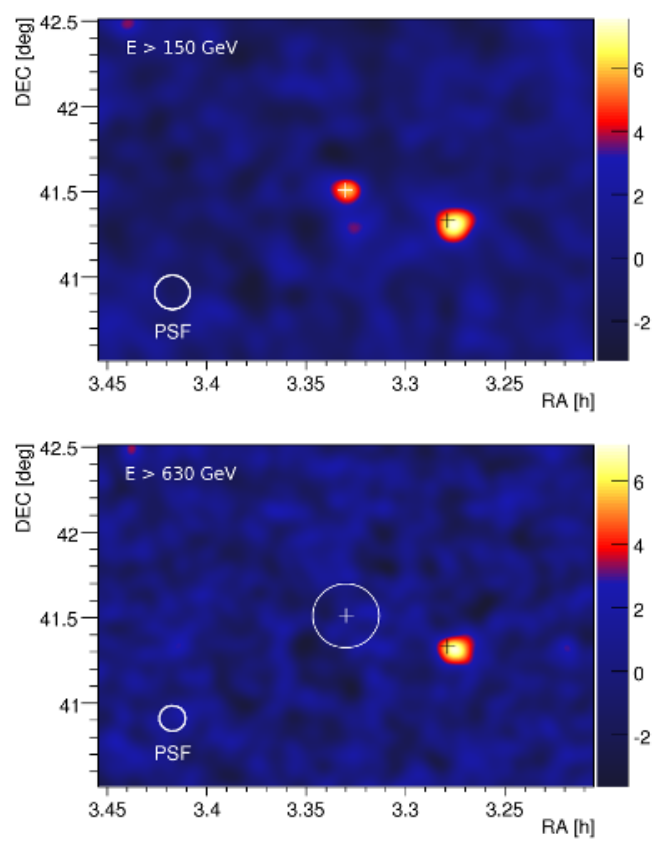

Figure 2. Significance sky map of the Perseus cluster, as observed with MAGIC [14], above $150 \mathrm{GeV}$ (top) and above 630 $\mathrm{GeV}$ (bottom). The white cross at the centre of the map marks the position of NGC 1275, the black cross IC 310, which is still visible above $630 \mathrm{GeV}$. The white circle shows the $0.15^{\circ}$ region used to compute the constraints mentioned in the text.

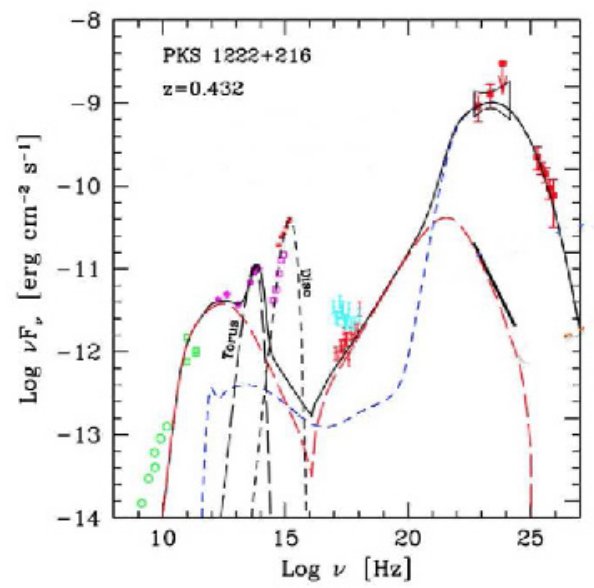

Figure 3. Spectral energy distribution of the flat spectrum radio quasar PKS 1222+216. See text for details.

from 3 to $400 \mathrm{GeV}$. The lack of a cut-off suggests that the emssion originates outside the broad line region, that results opaque to high-energy photons due to photon-photon absorption. On the other hand, the observed short variability of this source (of hour time scale) implies that the emitting region is rather small, hence located close to the central black hole. The spectral energy distribution and models of PKS $1222+216$ are shown in figure 3 .

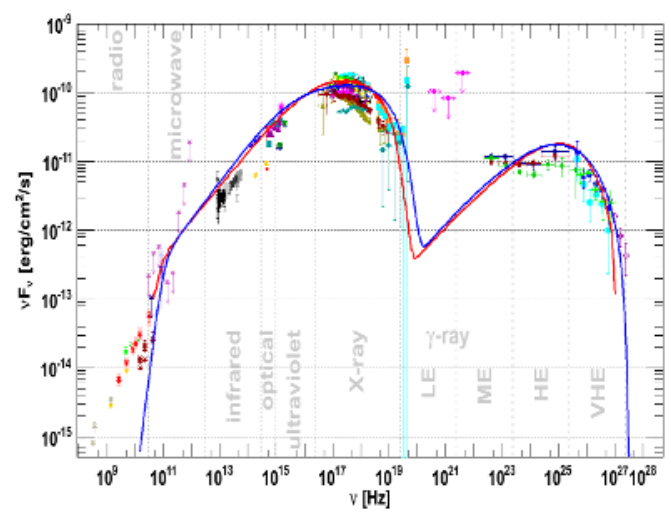

Figure 4. Spectral energy distribution of the blazar 1ES $1959+650$. The origin of the emission of this source cannot be explained with simple single zone models.

\subsection{Blazars whose emission model is uncertain}

There are some sources whose spectral energy distribution is not yet satisfactorily modeled. This includes the situation in which simple assumptions do not work (as in multi-zone models), and that in which no model, leptonic or hadronic, fits the observed spectrum. This is the situation for the blazar PKS 1424+240 [21], whose spectral energy distribution requires at least two different populations of leptons to be explained (a two-zone model). Another is the situation for 1ES 1959+650 [20] (figure 4), whose unusual plateau at high energies is not a feature of any leptonic model (based on inverse self Compton scattering). Hadronic models have failed to consistently model the source [20].

\section{Sources connected with galactic cosmic rays}

Supernova remnants are thought to be the sources of galactic cosmic rays, with energies up to about $10^{15} \mathrm{eV}$ [22]. The supposed acceleration mechanism in such objects is first order (diffuse shock) Fermi acceleration [23], which happens through scattering between upstream turbulence and the shock front (requiring a magnetized plasma upstream of the strong shock). This, being a process of electromagnetic nature, applies to both electrons and hadrons without differences. There is a class of supernova remnants surrounded by molecular clouds that is of particular interest for cosmic ray acceleration; in these sources, the radiation of $\gamma$ rays from hadrons can occur through protonnucleus interactions, and is related to a high number density of target nuclei in the molecular environment.

\subsection{Hints in favour of a hadronic component in W51C}

W51C is a supernova remnant with molecular cloud, associated with a giant molecular complex. The MAGIC observations of this source are shown in figure 5, for two 
energy ranges [26]. Both the morphology and the spectrum of this source indicate a possibly hadronic nature of the emission. The maximum of the emission coincides with interaction between the shock front and the molecular cloud in the nearby star forming region W51B, revealed as high velocity hydrohen (figure 5). The spectral energy distribution is best fit with a model having its main contribution from $\pi^{0}$ decay (figure 6).

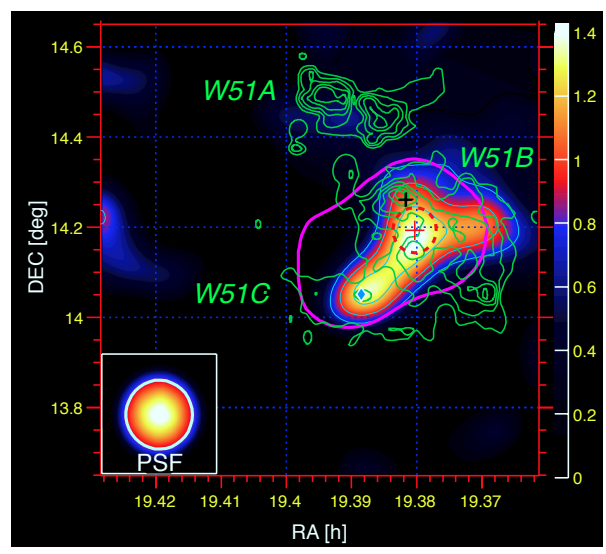

Figure 5. Relative flux map (excess/background) with MAGIC [26] (in colours), for energies larger than $1 \mathrm{TeV}$, overlaid with the region of shocked gas of the target molecular cloud [25] (red dashed ellipse), and the $21 \mathrm{~cm}$ radio continuum emission [24] (green contours). Pink contours mark the shape of the source first discovered by Fermi/LAT [27].

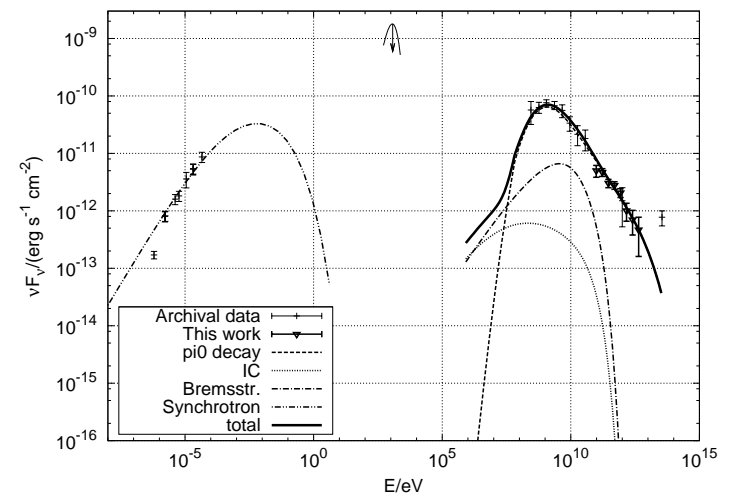

Figure 6. Spectral energy distribution of W51C, explained with a main hadronic contribution. $\gamma$ rays are produced from decays of neutral pions produced in proton-nucleus collisions. In the model shown here, the ratio of protons to electrons is 80:1. Simple leptonic models fail to fit the spectrum.

The non-spherical morphology of the $\gamma$ ray emission disfavours a scenario in which cosmic rays uniformly diffuse away from the shell [29]. Instead, those scenarios in which $\gamma$ are radiated in the shock regions, close to the acceleration site of their parent particles, which are more consistent with the high ionisation measurements in the cloud [28]. Re-acceleration of cosmic rays in the forward shock is not excluded [30].

\section{Electron Spectrum}

Cosmic ray electrons, whose propagation length is about $10^{5}$ light years, provide information about the diffuse spectrum of sources within a distance of order a few kiloparsecs. MAGIC measures cosmic ray electrons as standard background, as they initiate electromagnetic showers not correlated with the position of a source. Figure 7 shows the most recent MAGIC spectrum (in red), together with other existing measurements (in black), and the discrepancy of the observed electron spectrum from a pure power law that is supposed to describe the diffuse cosmic spectrum [31]. The origin of this contribution at high energy could be secondary electrons generated in interactions of cosmic rays with interstellar medium, or electrons from supernova explosions or pulsars. In exotic physics scenarios these are due to contributions from dark matter annihilation [31].

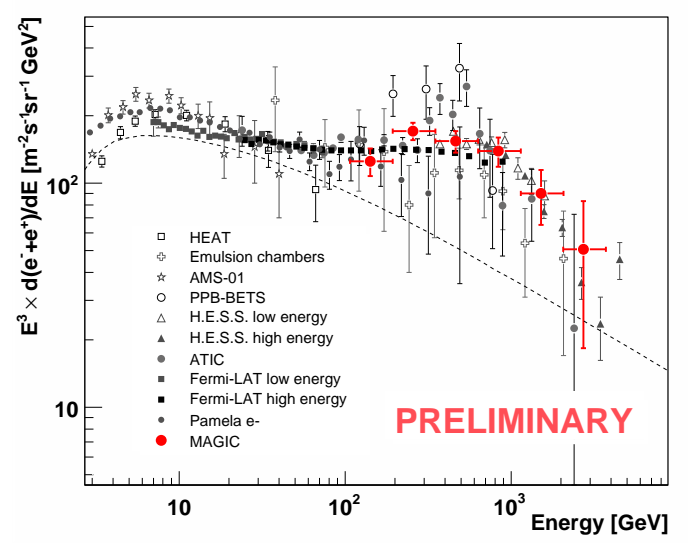

Figure 7. Electron spectrum measured with MAGIC (in red), overlaid with existing measurements [31]. The excess seen at high energy, still unexplained, could be due to secondary interactions of cosmic rays with the interstellar medium, supernova explosions, pulsars or dark matter contributions.

\section{Conclusions}

There are several classes of of sources detectable with $\gamma$ ray telescopes, that are suggested as possible cosmic ray accelerators: AGNs, radio galaxies, pulsars, supernova remnants. Extra-galactic sources appear point-like, so the only analysis that can be performed is the modelling of spectral energy distributions. Models based on accelerated hadrons provide a possible explanation for the emission of many AGNs. Some basic features of the accelerated particle spectra, as indices, breaks, cut-offs and normalisations, can be obtained from fits of energy distributions. In most models, the cosmic ray composition is averaged over protons and nuclei. It is not possible to constrain models for hadronic cross sections using only $\gamma$-ray data since the observations are indirect and the uncertainties on cross sections are folded with those on spectral parameters (shape, indices, normalisations), and on the source ambient (magnetic fields, target density). 
Hadron acceleration in sources would be confirmed with coordinated observation of neutrinos from decay of charged pions [6]. Coordination between $\gamma$-ray and neutrino telescopes has been suggested [32]. Neutrino events with coordinates close to preselected candidate sources are used to alert $\gamma$-ray observations. To date, only upper limits have been produced by the $\mathrm{TeV}$ observations.

\section{Acknowledgments}

We would like to thank the Instituto de Astrofísica de Canarias for the excellent working conditions at the Observatorio del Roque de los Muchachos in La Palma. The support of the German BMBF and MPG, the Italian INFN, the Swiss National Fund SNF, and the Spanish MICINN is gratefully acknowledged. This work was also supported by the CPAN CSD2007-00042 and MultiDark CSD2009-00064 projects of the Spanish ConsoliderIngenio 2010 programme, by grant DO02-353 of the Bulgarian NSF, by grant 127740 of the Academy of Finland, by the DFG Cluster of Excellence "Origin and Structure of the Universe", by the DFG Collaborative Research Centers SFB823/C4 and SFB876/C3, and by the Polish MNiSzW grant 745/N-HESS-MAGIC/2010/0.

\section{References}

[1] Mücke, A., Engel, R., Rachen, J. P., Protheroe, R. J., Stanev, T., Comp. Phys. Commun. 124, 290 (2000)

[2] Ahn, E.-J., Engel, R., Gaisser, T. K., Lipari, P., Stanev, T., Phys. Rev. D 80, 094003 (2009)

[3] Ostapchenko, S., Nuclear Physics B Proceedings Supplements, 151, 143-146 (2006)

[4] Kelner, S. R., Aharonian, F. A., Bugayov, V. V., Phys. Rev. D.74, 034018 (2006)

[5] Hillas, J. Phys. G31, R95 (2005)

[6] Kelner, S. R., Aharonian, F. A., Phys.Rev.D.78, 034013 (2008)

[7] Aleksić, J. et al for the MAGIC Collaboration, Astroparticle Physics, 35, 435 (2012)

[8] Pierre Auger Collaboration, Science, 318, 938 (2007)

[9] Urry, C. M. and Padovani, P., Publications of the Astronomical Society of the Pacific, 107, 803 (1995)

[10] Fermi-LAT and MAGIC, Aph. J. 736, 131 (2011)

[11] Fermi-LAT, MAGIC, VERITAS, Aph. J. 727, 129 (2011)
[12] Mücke, A., Protheroe, R. J., Engel, R., Rachen, J. P., Stanev, T., Astroparticle Physics 18, 593 (2003)

[13] Hillas A. M., Astroparticle Physics, 32, 160 (2009)

[14] Aleksić, J. et al for the MAGIC Collaboration, Astronomy \& Astrophysics, 541, 99A (2012)

[15] Aleksić, J. et al for the MAGIC Collaboration, Astronomy \& Astrophysics, 544, 96 (2012)

[16] Abramowski, A. et al. (HESS, MAGIC, VERITAS Collaboration), The Astrophysical Journal 746 (2012)

[17] Aleksić, J. et al for the MAGIC Collaboration, Science 320, 1752 (2008)

[18] Böttcher, M., Reimer, A., Marscher, A. P., The Astrophysical Journal 703, 1168 (2009)

[19] Stamerra, A. et al. (on behalf of the MAGIC collaboration), proceedings of the III Fermi Symposium (2011)

[20] Backes, M. (on behalf of the MAGIC collaboration), proceedings of the 5th International Symposium on High-Energy Gamma-Ray Astronomy, in press (2012)

[21] Prandini, E. (on behalf of the MAGIC collaboration), proceedings of the 5 th International Symposium on High-Energy Gamma-Ray Astronomy, in press (2012)

[22] Baade, W., Zwicky, F., Cosmic Rays from Supernovae, Proc. of the National Academy of Science (1934)

[23] Fermi, E., Physical Review, 75, 1169 (1949)

[24] Moon, D-S., Koo B-C., The Astrophysical Journal, 475 (1997)

[25] Carpenter, J.M. , Sanders, D.B., The Astronomical Journal, 116, 1856 (1998)

[26] Aleksić J. et al for the MAGIC Collaboration, Astronomy \& Astrophysics, 541 (2012)

[27] Abdo, A. A. et al, The Astrophysical Journal Letters, 706, L1 (2009)

[28] Ceccarelli C. et al, The Astrophysical Journal Letters, 740 (2011)

[29] Gabici, S., Aharonian, F. A., Casanova, S. Mon. Not. R. Astron. Soc., 396, 1629 (2009)

[30] Uchiyama, Y., Blandford R. D., Funk, S., Tajima, H., Tanaka, T. Astrophysical Journal Letters, 723, L122, (2010)

[31] Borla Tridon, D. (on behalf of the MAGIC collaboration), proceedings of the International Cosmic Ray Conference (ICRC) (2011)

[32] Ackermann, M., Bernardini, E., Galante, N., proceedings of the International Cosmic Ray Conference (ICRC) (2008) 